\title{
Progesterone receptor loss identifies luminal-type local advanced breast cancer with poor survival in patients who fail to achieve a pathological complete response to neoadjuvant chemotherapy
}

\author{
Sheng Chen ${ }^{1,2}$, Liang Huang ${ }^{1,2}$, Can-Ming Chen ${ }^{1,2}$ and Zhi-Ming Shao ${ }^{1,2,3}$ \\ ${ }^{1}$ Department of Breast Surgery, Fudan University Shanghai Cancer Center/Cancer Institute, Shanghai, P. R. China \\ 2 Department of Oncology, Shanghai Medical College, Fudan University, Shanghai, P. R. China \\ ${ }^{3}$ Institutes of Biomedical Science, Fudan University, Shanghai, P. R. China \\ Correspondence to: Sheng Chen, email:0456177@fudan.edu.cn \\ Zhi-Ming Shao, email: zhimingshao@yahoo.com \\ Keywords: breast cancer, neoadjuvant chemotherapy, survival, progesterone receptor, estrogen receptor \\ Received: March 28, $2015 \quad$ Accepted: May 13, $2015 \quad$ Published: May 22, 2015
}

This is an open-access article distributed under the terms of the Creative Commons Attribution License, which permits unrestricted use, distribution, and reproduction in any medium, provided the original author and source are credited.

\section{ABSTRACT}

The aim of this study was to investigate the potential of progesterone receptor $(\mathrm{PgR})$ as a biomarker for differentiating estrogen receptor (ER)-positive patients who fail to achieve a pathological complete response to neoadjuvant chemotherapy (NCT) with different prognoses. A total of 327 consecutive, locally advanced breast cancer patients with ER-positive disease were included in this study. According to their HER-2 and Ki-67 status, the patients were classified into the Luminal-A or Luminal-B subtype. We evaluated the clinical and pathological response to NCT and relapse or death occurring during follow-up according to PgR status in the different luminal subtypes. In the Luminal-B subtype, patients with PgR- tumors had a relatively higher pathological complete response (pCR) rate $(29.5 \%$ vs. $4.7 \%$ pCR, $P<0.001)$ and Miller-Payne grades (45.5\% vs. $23.5 \%$ of grade 4-5, $P=0033$ ) compared to PgR+ tumors. In Luminal-B patients with residual tumor after NCT, PgR loss was also independently correlated with poor relapse-free survival $(P=0.017 ; \mathrm{HR}=0.430 ; \mathrm{PgR}$ as a reference) and overall survival $(P=0.013 ; \mathrm{HR}=0.355 ; \mathrm{PgR}-$ as a reference $)$. However, in the Luminal-A subtype, there were no statistically significant differences between PgR+ and PgR- disease in response to NCT or survival. Our findings have demonstrated the prognostic value of PgR loss in the neoadjuvant setting, indicating that ER+/PgR- Luminal-B tumors warrant further attention due to their high risk of relapse after primary treatment.

\section{INTRODUCTION}

Neoadjuvant chemotherapy (NCT) is the standard of care for local control of local advanced breast cancer (LABC), and allowing breast conservation. Most studies confirmed that patients who achieved a pathological complete response (pCR) after NCT were expected to have a significantly more favorable outcome compared with patients with residual disease in the breast and/or axillary lymph nodes (known as non-pCR) [1, 2]. However, because only $10 \%-30 \%$ of patients experience pCR after primary treatment, the majority of patients still have a high risk of relapse and death. In the last decades, various approaches have focused on differentiating non-pCR responders with different outcomes. Several prognostic models have been reported, and some variables including node status, residual tumor size, Ki-67, hormonal receptor and human epidermal growth factor receptor-2 (HER2) have been shown to be potentially prognostic [3]. However, the results of these studies are not concordant, indicating that the prognostic value of some markers might not be consistent between different study populations.

In recent years, it has become widely accepted that 
breast cancer can be classified into multiple subtypes by immunohistochemical (IHC) analysis of the estrogen receptor (ER), progesterone receptor (PgR), HER-2, and $\mathrm{Ki}-67$. Such an analysis is considered to be a surrogate means for identifying molecular subtypes of breast cancer with different prognoses across treatment settings [4]. Many common subtypes have been identified, including two that are derived from hormonal receptor (HR)-positive tumors (Luminal-A and Luminal-B) and two that are derived from HR-negative tumors (triple-negative and HER-2+ cancers). The Luminal-type breast cancers, which are often associated with chemoresistance, have a better outcome compared with non-luminal-type breast cancers, as demonstrated by previous data $[5,6]$. Moreover, recent studies have suggested that in the neoadjuvant setting, failure to achieve a pCR is clearly associated with worse long-term outcomes in TNBC and HER-2+ breast cancer but not in the majority of hormone receptor-positive breast cancers $[7,8]$. Therefore, the difference in the biological features of $\mathrm{HR}+$ and HR- tumors has resulted in different recommendations for adjuvant systemic treatments and is often attributed to the heterogeneity of biomarker studies.

Compared with ER, PgR is often considered to be a weak prognostic marker for determining breast cancer subtype [9]. The absence of PgR may be associated with higher chemosensitivity and anti-estrogen resistance. Recent studies have indicated that ER+, PgR- tumors had more aggressive features that resulted in worse outcomes compared with ER+, PgR+ tumors [10]. However, the prognostic value of $\mathrm{PgR}$ in the neoadjuvant setting remains controversial. In the present study, we investigated the relationship between PgR expression and long-term survival in Luminal-type breast cancer patients who received NCT, aiming to identify a distinct subset of $\mathrm{ER}+$ tumors.

\section{RESULTS}

\section{Patient characteristics and response to NCT}

The median age of the 327 patients was 49 years (range: 25-70 years). All patients were diagnosed with stage II or III disease, and $41.0 \%$ of them were postmenopausal at diagnosis. A total of 193 patients received an anthracycline-based regimen, 114 received a taxanebased regimen, and 20 received both anthracycline
A

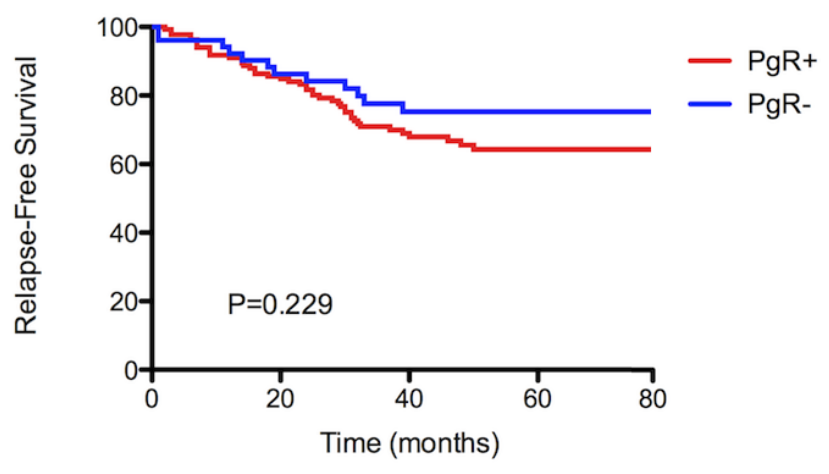

C

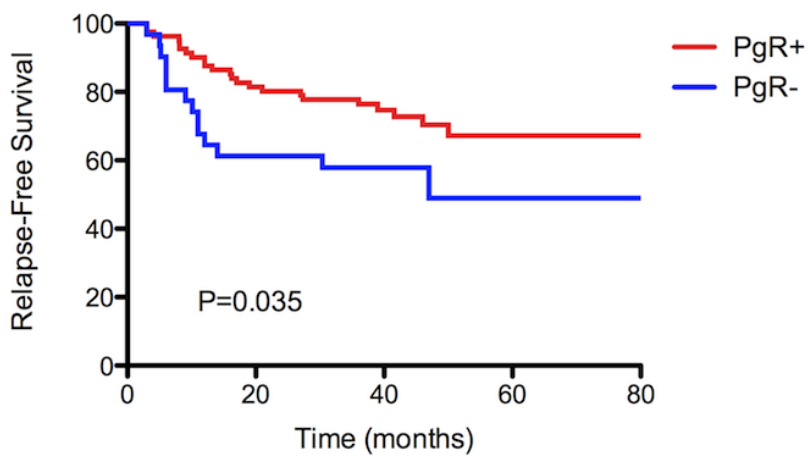

B

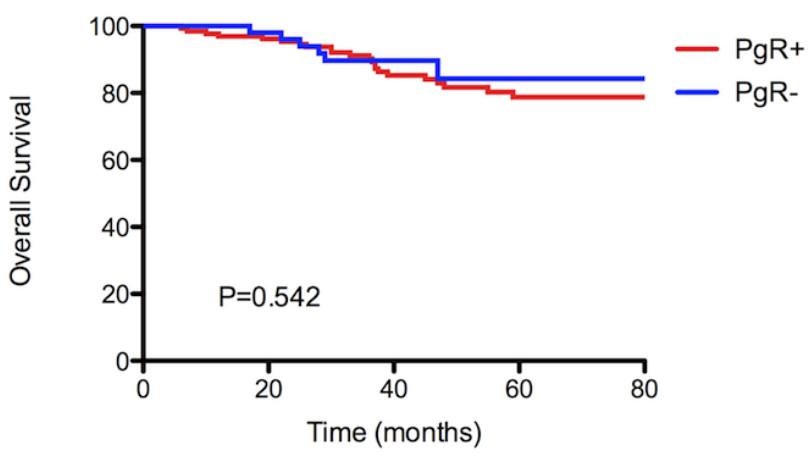

$\mathrm{D}$

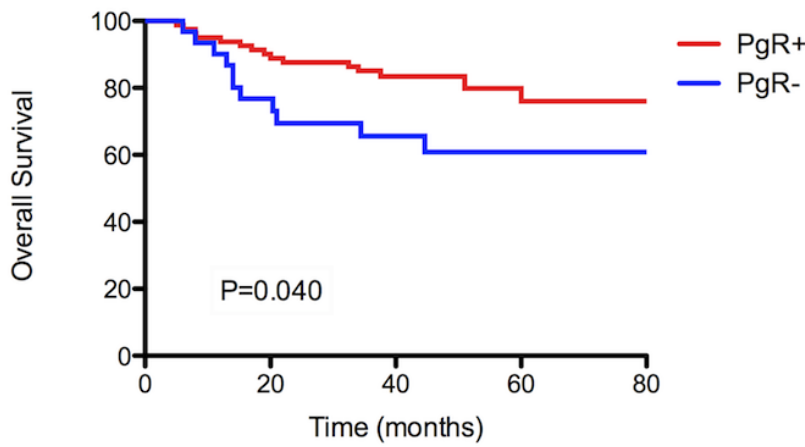

Figure 1: Kaplan-Meier survival curves for relapse-free survival (RFS) and overall survival (OS) of non-pCR patients with Luminal-A (A, B) and Luminal-B (C, D) primary tumors by PgR status. PgR loss was significantly correlated with poor survival (both RFS and OS) in Luminal-B patients $(P=0.035$ and $P=0.040$, respectively), but not in Luminal-A patients $(P=0.229$ and $P=0.542$, respectively). 
Table 1: Characteristics of $327 \mathrm{ER}+$ patients by breast cancer subtype.

\begin{tabular}{|c|c|c|c|c|c|c|c|}
\hline \multirow[t]{2}{*}{ Characteristics } & \multicolumn{2}{|c|}{$\begin{array}{c}\text { Total } \\
(\mathbf{N}=327)\end{array}$} & \multicolumn{2}{|c|}{$\begin{array}{c}\text { Lumina-A } \\
(N=198)\end{array}$} & \multicolumn{2}{|c|}{$\begin{array}{c}\text { Luminal-B } \\
(\mathrm{N}=129)\end{array}$} & \multirow[t]{2}{*}{ P value } \\
\hline & No. & $\%$ & No. & $\%$ & No. & $\%$ & \\
\hline Patient age, years & & & & & & & 0.737 \\
\hline$<50$ & 166 & 50.8 & 102 & 51.5 & 64 & 49.6 & \\
\hline$\geq 50$ & 161 & 49.2 & 96 & 48.5 & 65 & 50.4 & \\
\hline Menopausal status & & & & & & & 0.975 \\
\hline Pre & 193 & 59.0 & 117 & 59.1 & 76 & 58.9 & \\
\hline Post & 134 & 41.0 & 81 & 40.9 & 53 & 41.1 & \\
\hline Tumor stage at diagnosis & & & & & & & 0.001 \\
\hline $\mathrm{T} 2$ & 116 & 35.5 & 76 & 38.4 & 40 & 31.0 & \\
\hline $\mathrm{T} 3$ & 156 & 47.7 & 101 & 51.0 & 55 & 42.6 & \\
\hline $\mathrm{T} 4$ & 55 & 16.8 & 21 & 10.6 & 34 & 26.4 & \\
\hline LN status at diagnosis & & & & & & & 0.609 \\
\hline- & 81 & 24.8 & 51 & 25.8 & 30 & 23.3 & \\
\hline+ & 246 & 75.2 & 147 & 74.2 & 99 & 76.7 & \\
\hline PgR status & & & & & & & 0.358 \\
\hline- & 102 & 31.2 & 58 & 29.3 & 44 & 34.1 & \\
\hline+ & 225 & 68.8 & 140 & 70.7 & 85 & 65.9 & \\
\hline NCT regimen & & & & & & & 0.038 \\
\hline Anthracycline-based & 198 & 60.6 & 130 & 65.7 & 68 & 52.7 & \\
\hline Taxane-based & 103 & 31.5 & 52 & 26.3 & 51 & 39.5 & \\
\hline Anthracycline and & 26 & 8.0 & 16 & 8.1 & 10 & 7.8 & \\
\hline Taxane & & & & & & & \\
\hline Clinical response & & & & & & & 0.931 \\
\hline $\mathrm{CR}$ & 35 & 10.7 & 21 & 10.6 & 14 & 10.9 & \\
\hline PR & 174 & 53.2 & 107 & 54.0 & 67 & 51.9 & \\
\hline $\mathrm{SD} / \mathrm{PD}$ & 118 & 36.1 & 70 & 35.4 & 48 & 37.2 & \\
\hline Pathological response & & & & & & & 0.009 \\
\hline $\mathrm{pCR}$ & 27 & 8.3 & 10 & 5.1 & 17 & 13.2 & \\
\hline Non-pCR & 300 & 91.7 & 188 & 94.9 & 112 & 86.8 & \\
\hline
\end{tabular}

\section{Abbreviations: LN, lymph node; PgR, progesterone receptor; NCT, neoadjuvant chemotherapy; $\mathrm{CR}$, complete response; $\mathrm{PR}$, partial response; $\mathrm{SD} / \mathrm{PD}$, stable disease or progression disease;}

and taxane as an NCT regimen. All the patients had confirmed ER+ disease before NCT, and 225 of them also had positive PgR expression in their primary tumors. According to the expression of HER-2 and Ki-67 in CNB tumor samples as determined by IHC analysis, 198 patients were classified into the Luminal-A subtype, whereas 129 patients were classified into the Luminal-B subtype (56 with HER-2+, 73 with HER-2-). The patient characteristics and responses to NCT by breast cancer subtype are shown in Table 1. Remarkably, patients with Luminal-B disease were more likely to achieve a pCR after NCT, with an observed pCR rate of $13.2 \%$; on the other hand, patients with Luminal-A disease had an observed pCR rate of $5.1 \%(P=0.009)$.

Furthermore, in Table 2 we show the distributions of patient responses to NCT among the different subtypes, demonstrating the relationship between PgR and treatment response. In the Luminal-A subtype, there were no statistically significant differences between $\mathrm{PgR}+$ and $\mathrm{PgR}$ - disease with respect to clinical response, pathological response according to $\mathrm{pCR}$ rate, or response scores according to the MP grading system. However, in the Luminal-B subtype, patients with PgR- tumors had a relatively higher pCR rate $(29.5 \%$ vs. $4.7 \%$ pCR, $P<$ $0.001)$ and MP grades (45.5\% vs. $23.5 \%$ grade $4-5, P=$ 0033).

\section{Correlation between PgR and survival}

Overall, the observed 5-year RFS and 5-year OS of the 327 patients were $68 \%$ and $81 \%$, respectively, in the 
Table 2 Patient responses to NCT according to PgR status

\begin{tabular}{|c|c|c|c|c|c|c|}
\hline \multirow[t]{2}{*}{ Response } & \multicolumn{3}{|c|}{ Luminal-A $(n=198)$} & \multicolumn{3}{|c|}{ Luminal-B $(n=129)$} \\
\hline & ALL & PgR- & PgR+ & ALL & PgR- & PgR+ \\
\hline \multicolumn{7}{|l|}{ Clinical response } \\
\hline CR & $21(10.6 \%)$ & $8(13.8 \%)$ & $13(9.3 \%)$ & $14(10.9 \%)$ & $7(15.9 \%)$ & $7(8.2 \%)$ \\
\hline \multirow[t]{2}{*}{ PR } & 107 & $33(56.9 \%)$ & $74(52.9 \%)$ & $67(51.9 \%)$ & $23(52.3 \%)$ & $44(51.8 \%)$ \\
\hline & $(54.0 \%)$ & & & & & \\
\hline SD/PD & $70(35.4 \%)$ & $17(29.3)$ & $53(37.9 \%)$ & $48(37.2 \%)$ & $14(31.8 \%)$ & $34(40.0 \%)$ \\
\hline P value & \multicolumn{3}{|c|}{0.415} & & \multicolumn{2}{|c|}{0.365} \\
\hline \multicolumn{7}{|c|}{ Pathological response } \\
\hline pCR & $10(5.1 \%)$ & $6(10.3 \%)$ & $4(2.9 \%)$ & $17(13.2 \%)$ & $13(29.5 \%)$ & $4(4.7 \%)$ \\
\hline \multirow[t]{2}{*}{ Non-pCR } & 188 & $52(89.7 \%)$ & 136 & $112(86.8 \%)$ & $31(70.5 \%)$ & $81(95.3 \%)$ \\
\hline & \multicolumn{2}{|l|}{$(94.9 \%)$} & \multicolumn{2}{|l|}{$(97.1 \%)$} & & \\
\hline P value & \multicolumn{3}{|c|}{$0.067 *$} & & \multicolumn{2}{|c|}{$<0.001$} \\
\hline \multicolumn{7}{|l|}{ MP grading } \\
\hline $5-4$ & $34(17.2 \%)$ & $13(22.4)$ & $21(15.0 \%)$ & $40(31.0 \%)$ & $20(45.5 \%)$ & $20(23.5 \%)$ \\
\hline \multirow[t]{2}{*}{3} & 105 & $31(53.4 \%)$ & $74(52.9 \%)$ & $48(37.2 \%)$ & $14(31.8 \%)$ & $34(40.0 \%)$ \\
\hline & $(53.0 \%)$ & & & & & \\
\hline $2-1$ & $59(29.8 \%)$ & $14(24.1 \%)$ & $45(32.1 \%)$ & $41(31.8 \%)$ & $10(22.7 \%)$ & $31(36.5 \%)$ \\
\hline$P$ value & & & 33 & & & 33 \\
\hline
\end{tabular}

Abbreviations: NCT, neoadjuvant chemotherapy; PgR, progesterone receptor; $C R$, complete response;

PR, partial response; SD/PD, stable disease or progression disease; MP, Miller-Payne;

* Fisher's exact test

A

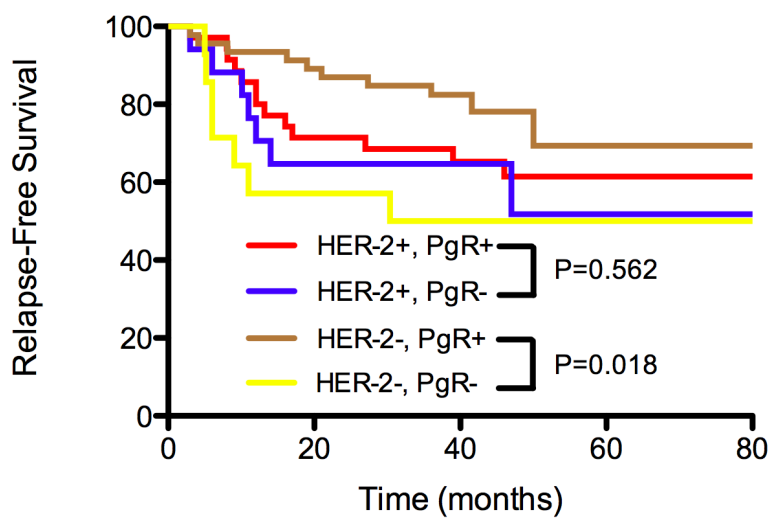

B

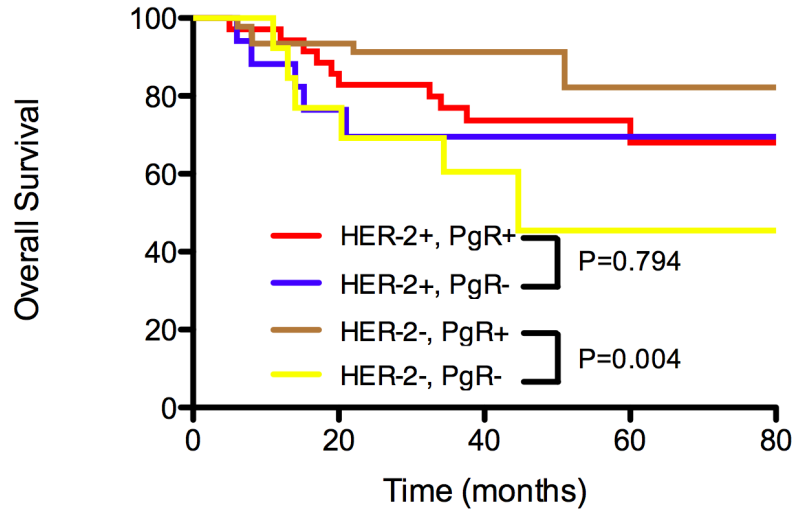

Figure 2: Kaplan-Meier survival curves for relapse-free survival (RFS) and overall survival (OS) by PgR status and HER2 status in non-pCR patients with Luminal-B primary tumors. PgR was significantly correlated with RFS and OS in HER-2Luminal-B patients $(P=0.018$ and $P=0.004$, respectively) but not in HER-2+ Luminal-B patients $(P=0.562$ and $P=0.794$, respectively). 
Table 3 Univariate and multivariate survival analysis for Luminal-B patients with residual disease after NCT

\begin{tabular}{|c|c|c|c|c|c|c|c|c|}
\hline \multirow{3}{*}{ Variable } & \multicolumn{4}{|c|}{ RFS } & \multicolumn{4}{|c|}{ OS } \\
\hline & \multirow{2}{*}{$\frac{\text { Unv. }}{P}$} & \multicolumn{3}{|c|}{ Muv. } & \multirow{2}{*}{$\begin{array}{c}\text { Unv. } \\
P\end{array}$} & \multicolumn{3}{|c|}{ Muv. } \\
\hline & & $\begin{array}{l}\text { Harzard } \\
\text { ratio }\end{array}$ & $95 \% \mathrm{CI}$ & $\mathbf{P}$ & & Hazard ratio & $95 \% \mathrm{CI}$ & $\mathbf{P}$ \\
\hline $\begin{array}{l}\text { Patient age, years } \\
\quad<50 \\
\geq 50\end{array}$ & 0.153 & - & - & - & 0.371 & - & - & - \\
\hline $\begin{array}{l}\text { Menopausal status } \\
\text { Pre } \\
\text { Post }\end{array}$ & 0.419 & - & - & - & 0.707 & - & - & - \\
\hline $\begin{array}{l}\text { NCT regimen } \\
\text { Anthracycline-based } \\
\text { Taxane-based } \\
\text { Anthracycline and Taxane }\end{array}$ & 0.281 & - & - & - & 0.532 & - & - & - \\
\hline $\begin{array}{l}\text { Primary tumor stage } \\
\text { T2 } \\
\text { T3 } \\
\text { T4 }\end{array}$ & 0.108 & - & - & - & 0.107 & - & - & - \\
\hline $\begin{array}{l}\text { Primary LN status } \\
- \\
+\end{array}$ & 0.123 & - & - & - & 0.067 & - & - & - \\
\hline $\begin{array}{c}\text { PgR status } \\
- \\
+\end{array}$ & 0.035 & $\begin{array}{l}\text { Ref. } \\
0.430\end{array}$ & $0.215-0.858$ & 0.017 & 0.040 & $\begin{array}{c}\text { Ref. } \\
0.355\end{array}$ & $0.157-0.801$ & 0.013 \\
\hline $\begin{array}{l}\text { HER-2 status } \\
\quad- \\
+\end{array}$ & 0.275 & - & - & - & 0.141 & - & - & - \\
\hline $\begin{array}{l}\text { Residual tumor }(\mathrm{cm}) \\
\quad<2 \\
2-5 \\
>5\end{array}$ & 0.003 & $\begin{array}{l}\text { Ref. } \\
1.690 \\
3.090\end{array}$ & $\begin{array}{l}0.727-3.931 \\
1.274-7.491\end{array}$ & 0.046 & 0.004 & $\begin{array}{l}\text { Ref. } \\
1.559 \\
3.664\end{array}$ & $\begin{array}{c}0.540-4.499 \\
1.276-10.519\end{array}$ & 0.041 \\
\hline $\begin{array}{l}\text { Residual LN involvement } \\
0 \\
1-3 \\
4+\end{array}$ & 0.010 & $\begin{array}{l}\text { Ref. } \\
0.843 \\
2.624\end{array}$ & $\begin{array}{l}0.233-3.049 \\
0.893-7.712\end{array}$ & 0.013 & 0.014 & $\begin{array}{l}\text { Ref. } \\
0.949 \\
3.408\end{array}$ & $\begin{array}{c}0.152-5.929 \\
0.769-15.096\end{array}$ & 0.027 \\
\hline $\begin{array}{l}\text { Residual tumor grade } \\
\text { I-II } \\
\text { III }\end{array}$ & 0.027 & - & - & 0.659 & 0.175 & - & - & - \\
\hline $\begin{array}{l}\text { Vascular invasion } \\
\text { Yes } \\
\text { No }\end{array}$ & 0.623 & - & - & - & 0.851 & - & - & - \\
\hline $\begin{array}{l}\text { Residual tumor } \mathrm{Ki}-67 \\
\quad<15 \% \\
\quad \geq 15 \%\end{array}$ & 0.011 & $\begin{array}{l}\text { Ref. } \\
2.245\end{array}$ & $1.172-4.298$ & 0.015 & 0.403 & - & - & - \\
\hline $\begin{array}{l}\text { CT } \\
\text { Anthracycline } \\
\text { Taxane } \\
\text { Anthracycline and Taxane } \\
\text { None }\end{array}$ & 0.619 & - & - & - & 0.258 & - & - & - \\
\hline $\begin{array}{l}\text { RT } \\
\text { Yes } \\
\text { No }\end{array}$ & 0.372 & - & - & - & 0.200 & - & - & - \\
\hline $\begin{array}{ll}\text { ET } & \\
& \text { Yes } \\
& \text { No }\end{array}$ & 0.611 & - & - & - & 0.359 & - & - & - \\
\hline
\end{tabular}

Abbreviations: Unv, univariate analysis; Muv, multivariate analysis; Ref, reference; LN, lymph node; NCT, neoadjuvant chemotherapy; PgR, progesterone receptor; HER-2, human epidermal receptor-2; CT, chemotherapy; RT, radiotherapy; ET, endocrinotherapy

Luminal-A subtype and $67 \%$ and $80 \%$, respectively, in the Luminal-B subtype. Failure to achieve a pCR is clearly associated with worse long-term outcomes in Luminal-B breast cancer, although this negative prognostic association is not statistically significant in Luminal-A breast cancers. The 5-year RFS and 5-year OS of patients with Luminal-A patients with pCR were $90.0 \%$ (compared with $67.5 \%$ in non-pCR patients, log-rank test $P=0.168$ ) and $90.0 \%$ (compared with $80.4 \%$ in non-pCR patients, log-rank test $P=0.476$ ), respectively, whereas the 5-year RFS and 5-year OS of Luminal-B patients with pCR were $94.1 \%$ (compared with $61.4 \%$ in non-pCR patients, logrank test $P=0.018$ ) and $100.0 \%$ (compared with $75.9 \%$ in non-pCR patients, log-rank test $P=0.023$ ), respectively.

In non-pCR responders, univariate survival analyses were performed separately in the Luminal-A 
and Luminal-B categories to detect the prognostic value of PgR. PgR loss was significantly correlated with poor survival (both RFS and OS) in Luminal-B patients but not in Luminal-A patients. The distributions of the survival curves are shown in Figure 1A-1D. Additionally, multivariate survival analysis using the Cox regression model was performed in Luminal-B patients. PgR status $(P=0.017 ; \mathrm{HR}=0.430 ; \mathrm{PgR}-$ as a reference $)$, residual tumor size $(P=0.003$; HR $=1.690$ for $2-5 \mathrm{~cm}, \mathrm{HR}=3.090$ for $>5 \mathrm{~cm} ;<2 \mathrm{~cm}$ as a reference), residual lymph node involvement $(P=0.014$; HR $=0.843$ for $1-3$ positive node, $\mathrm{HR}=2.624$ for $\geq 4$ positive node; 0 positive node as a reference), and $\mathrm{Ki}-67(P=0.015 ; \mathrm{HR}=2.245 ;<15 \%$ as a reference) were independent prognostic variables for RFS. PgR status $(P=0.013 ; \mathrm{HR}=0.355 ; \mathrm{PgR}$ - as a reference $)$, residual tumor size $(P=0.041 ; \mathrm{HR}=1.559$ for $2-5 \mathrm{~cm}$, $\mathrm{HR}=3.664$ for $>5 \mathrm{~cm} ;<2 \mathrm{~cm}$ as a reference), and residual lymph node involvement $(P=0.014$; HR $=0.949$ for $1-3$ positive node, $\mathrm{HR}=3.408$ for $\geq 4$ positive node; 0 positive node as a reference) were also independent prognostic variables for OS. The results of the univariate and multivariate survival analyses are shown in Table 3.

In addition, PgR had different prognostic values for patient survival with respect to HER-2 status (Figure 2A, 2B). It was significantly correlated with RFS and OS in HER-2- Luminal-B patients $(P=0.018$ and $P=0.004$, respectively). However, in HER-2+ Luminal-B patients, there was no significant difference in either RFS or OS with respect to the PgR category $(P=0.562$ and $P=0.794$, respectively).

\section{DISCUSSION}

NCT, which downstages the disease and enables surgery to be performed in those initially deemed inoperable, has been widely accepted as a standard of care for LABCs. Most published studies have affirmed that patients who achieve pCR, irrespective of the initial stage and molecular subtype, have a very low risk of relapse and death $[2,11]$. However, a pCR occurs in only approximately $10 \%-30 \%$ of all cases and depends on the biological features of the primary tumor to a large extent. For patients who fail to achieve pCR, the response to subsequent treatment and prognosis also varies between different subgroups $[12,13]$.

Although gene expression profiling has led to a better understanding of the biological phenotypes of breast cancer, its technical complexity and high costs have limited its clinical application. The combination of IHC markers, including ER, PgR, HER-2, and Ki-67, has been accepted as a substitute for molecular subtypes, although the results obtained with gene expression profiling and IHC do not exactly correspond $[14,15]$. It is well known that HER-2 overexpression, negativity for hormonal receptors, and a high Ki-67 index are significantly correlated with high chemosensitivity and result in different responses to chemotherapy [16]. Therefore, breast cancer subtypes as defined by differential ER, PgR, HER-2, and Ki-67 staining may display different response rates and different prognoses in the neoadjuvant setting. For ER+ breast cancers, neoadjuvant endocrine therapy other than chemotherapy might be a more appropriate choice for Luminal-A LABCs; however, there is a lack of consensus on the threshold indication for inclusion of NCT for the Luminal-B subtype. Compared to Luminal-A breast cancer, the Luminal-B phenotype has aggressive clinical and biological features and is characterized by lower levels of ER-related genes, higher levels of HER2-associated genes, and higher levels of proliferative markers $[17,18]$.

Given that HER-2 overexpression was only observed in $30 \%$ of Luminal-B tumors, the Luminal-B phenotype still represents a heterogeneous group of breast cancers. A better understanding of this tumor phenotype might allow better determination of appropriate diagnostic and therapeutic strategies. Several approaches have been undertaken to differentiate Luminal-B tumors with different chemosensitivity and survival $[19,20]$. Recently, G. Cancello [21] reported a relatively good prognosis in the PgR + subgroup of Luminal-B patients and highlighted the significant impact of PgR status on the outcome of patients with early breast cancer. The author claimed to consider more chemotherapy in the ER+/PgR-/HER-2subgroup and less radical therapy for the "triple positive" subgroup in the primary setting. It is speculated that ER+/ PgR- tumors may display more aggressive features than $\mathrm{ER}+/ \mathrm{PgR}+$ tumors. In this study, we also found that $\mathrm{PgR}$ was an important predictive and prognostic biomarker in Luminal-B patients in the neoadjuvant setting. Compared with $\mathrm{PgR}+$ patients, patients with PgR- Luminal-B disease had relatively higher $\mathrm{pCR}$ rates and MP grades. However, among Luminal-B patients failing to achieve pCR, the loss of $\mathrm{PgR}$ resulted in a high risk of relapse or death. This paradox indicated that PgR status could be used to identify a unique phenotype of Luminal-B breast cancers. This group of tumors has higher response rates to neoadjuvant chemotherapy; however, this advantage is not clearly translated into survival benefit among non-pCR patients.

In this study, the discrimination of Luminal-A and Luminal-B subtype were based on the criteria of St.Gallen Consensus 2011. However, in 2013, some experts suggested that ER+/PgR-low patients should also be included in the Luminal-B subtype based on gene expression profiling. Interestingly, in this study, PgR was only prognostic in HER-2-/Luminal-B tumors but not in HER-2+/Luminal-B or Luminal-A ER+ tumors, which indicated that PgR-/HER-2- with low Ki-67 could not be simply affiliated to Luminal-B subtype.

Considering their high response rate to chemotherapy and poor survival, ER+/PR-/HER-2patients with high proliferation rates displayed biological behaviors similar to those of patients with triple- 
negative breast cancer [12]. Despite a better response to neoadjuvant treatments compared to other subtypes of tumors, the long-term prognosis of PR-/HER-2Luminal-B patients is worse overall than that of other subsets, particularly in the first three years after treatment (Figure 2). Thus, Luminal-B tumors that are resistant to primary chemotherapy might exhibit aggressive behavior and benefit little from further chemo-endocrine treatment.

The loss of PgR may be a surrogate marker of a nonfunctional ER, and it also reflects aberrant growth factor signaling that activates other ER functions other than the PgR pathway [22]. Thus, lack of PgR may contribute to endocrine resistance, which results in poor outcome. Furthermore, ER+/PgR- tumors might also express higher levels of HER-1 and HER-2, which are associated with a significantly poorer outcome, whereas in $\mathrm{ER}+/ \mathrm{PgR}+$ disease, neither HER-1 expression nor HER-2 overexpression is associated with outcome [22]. In this study, PgR failed to show prognostic value in HER$2+$ patients. Because trastuzumab was not given prior to surgery in this study, HER-2+ patients who did not achieve pCR could still benefit from adjuvant trastuzumab, which might result in an unclear survival impact of PgR loss in this specific phenotype. Thus, additional studies examining ER+/HER-2+ patients treated with trastuzumab prior to surgery are needed to clarify this issue.

In summary, although Luminal-type patients are assumed to have a relatively good prognosis, different permutations and combinations of biomarkers are associated with variable tumor behavior, as we have described for the ER+/PR-/HER-2- subset. Our findings have demonstrated the prognostic value of PgR loss in the neoadjuvant setting, indicating that PgR- high Luminal-B tumors require greater attention due to their high risk of relapse after primary treatment. Prospective approaches regarding tailored treatment strategies should be considered for this unique subset of Luminal-B tumors.

\section{PATIENTS AND METHODS}

\section{Study population}

The patient cohort in the present study had ER+ primary breast cancer and received NCT followed by surgery at Shanghai Cancer Center from 1999 to 2009. All patients' diseases were confirmed as invasive carcinoma through core needle biopsy (CNB), and node status was assessed through fine needle aspiration (FNA) of palpable lymph nodes before NCT. Patients who had any treatment prior to NCT were not eligible for this study. Other exclusion criteria included metastatic disease before surgery, bilateral breast cancer, male breast cancer, and inflammatory breast cancer. Informed written consent was obtained from all subjects prior to the study.
A total of 327 consecutive locally advanced breast cancer patients met the above criteria. The NCT regimens included anthracycline-based, taxane-based, and anthracycline-taxane-based regimens for a median of 3 cycles, as previously reported [23]. For all patients, the surgical procedure included mastectomy and axillary lymph node dissection upon the completion of NCT. Additional cycles of chemotherapy were subsequently performed to complete a total of 6-8 cycles followed by radiation therapy at the discretion of the treating physician and radiologist. Endocrine therapy was recommended for all patients in the present study. Due to health insurancerelated limitations, trastuzumab was not utilized before surgery in patients overexpressing HER-2.

Responses to NCT were evaluated clinically and pathologically. Clinical response was based on the reduction of tumor size and node status as detected through MRI and ultrasound according to Response Evaluation Criteria in Solid Tumors (RECIST) 1.1 [24]. Pathological response was evaluated according to the Miller-Payne (MP) grading system [25]. A pathological complete response (pCR) after NCT was defined as the absence of invasive carcinoma in both the breast tissue (MP Grade 5) and lymph nodes of the resected specimen. Patients with ductal carcinoma in situ (DCIS) only were also considered as pCR responders.

\section{Immunohistochemistry and intrinsic subtypes}

Immunohistochemistry and/or fluorescence in situ hybridization (FISH) assays were used for the detection of ER, PgR, HER-2 and Ki-67. The cut-off value for ER and PgR positivity was $1 \%$ tumor cells with positive nuclear staining. HER-2 was evaluated as $0,1+, 2+$ or $3+$ using circumferential membrane-bound staining, and positivity (HER-2+) was considered as $3+$ based a positive result via IHC analysis or positivity via FISH; cases considered as 0 to $1+$ or $2+$ without FISH positivity were regarded as negative (HER-2-). The Ki-67 value was expressed as the percentage of positive cells (at least 1000) with nuclear staining in each case. The antibodies used were as follows: ER (M7047, clone 1D5, Dako, Denmark), PgR (M3569, clone PgR 636, Dako), HER-2 (A0485, polyclonal rabbit antibody, Dako), and Ki-67 (M7240, clone MIB-1, Dako). On the basis of the 2011 St. Gallen consensus [15], the patient cohort of the present study was divided into two intrinsic subtypes: Luminal-A (HER-2 negative and Ki$67<15 \%$ ) and Luminal-B (HER-2 positive, or HER-2 negative and $\mathrm{Ki}-67 \geq 15 \%$ ).

\section{Statistical analysis}

The chi-square test was used to evaluate the distribution of patient characteristics and the relationship between PgR and response to NCT among Luminal-A and 
Luminal-B subtypes. The Fisher exact test was performed when necessary. Survival analyses were performed using the Cox regression model. Statistically significant prognostic variables in the univariate analysis were tested in the multivariate model with forward selection. The distributions of survival curves were shown using the Kaplan-Meier method, and the differences were measured using the log-rank test. Relapse-free survival (RFS) was calculated from the date of surgery to the date of disease relapse (local or distant relapse or death from any cause). Overall survival (OS) was calculated from the date of diagnosis to the date of death or last follow-up. Patients without events or death were censored at the last follow-up. All P-values reported were two sided and were calculated at a significance level of 0.05. All statistical procedures were carried out using SPSS (version 13.0; SPSS Company, Chicago, IL).

\section{ACKNOWLEDGMENTS AND GRANT SUPPORT}

This research was supported by the National Natural Science Foundation of China (81302298). The funders had no role in the study design, data collection and analysis, decision to publish, or preparation of the manuscript.

\section{CONFLICTS OF INTEREST}

The authors declare no potential conflicts of interest, and there are no financial disclosures.

\section{REFERENCES}

1. Bonnefoi H, Litiere S, Piccart M, Macgrogan G, Fumoleau P, Brain E, Petit T, Rouanet P, Jassem J, Moldovan C, Bodmer A, Zaman K, Cufer T, Campone M, Luporsi E, Malmstrom $\mathrm{P}$, et al. Pathological complete response after neoadjuvant chemotherapy is an independent predictive factor irrespective of simplified breast cancer intrinsic subtypes: a landmark and two-step approach analyses from the EORTC 10994/BIG 1-00 phase III trial. Ann Oncol. 2014; 25: 1128-36.

2. Rastogi P, Anderson SJ, Bear HD, Geyer CE, Kahlenberg MS, Robidoux A, Margolese RG, Hoehn JL, Vogel VG, Dakhil SR, Tamkus D, King KM, Pajon ER, Wright MJ, Robert J, Paik S, et al. Preoperative chemotherapy: updates of National Surgical Adjuvant Breast and Bowel Project Protocols B-18 and B-27. J Clin Oncol. 2008; 26:778-785.

3. Colleoni M, Bagnardi V, Rotmensz N, Dellapasqua S, Viale G, Pruneri G, Veronesi P, Torrisi R, Luini A, Intra M, Galimberti V, Montagna E and Goldhirsch A. A risk score to predict disease-free survival in patients not achieving a pathological complete remission after preoperative chemotherapy for breast cancer. Ann Oncol. 2009; 20:11781184.
4. Sorlie T, Tibshirani R, Parker J, Hastie T, Marron JS, Nobel A, Deng S, Johnsen H, Pesich R, Geisler S, Demeter J, Perou CM, Lonning PE, Brown PO, Borresen-Dale AL and Botstein D. Repeated observation of breast tumor subtypes in independent gene expression data sets. Proc Natl Acad Sci U S A. 2003; 100:8418-8423.

5. Oh DS, Troester MA, Usary J, Hu Z, He X, Fan C, Wu J, Carey LA and Perou CM. Estrogen-regulated genes predict survival in hormone receptor-positive breast cancers. J Clin Oncol. 2006; 24:1656-1664.

6. Perou CM, Sorlie T, Eisen MB, van de Rijn M, Jeffrey SS, Rees CA, Pollack JR, Ross DT, Johnsen H, Akslen LA, Fluge O, Pergamenschikov A, Williams C, Zhu SX, Lonning PE, Borresen-Dale AL, et al. Molecular portraits of human breast tumours. Nature. 2000; 406:747-752.

7. von Minckwitz G, Untch M, Blohmer JU, Costa SD, Eidtmann H, Fasching PA, Gerber B, Eiermann W, Hilfrich J, Huober J, Jackisch C, Kaufmann M, Konecny GE, Denkert C, Nekljudova V, Mehta K, et al. Definition and impact of pathologic complete response on prognosis after neoadjuvant chemotherapy in various intrinsic breast cancer subtypes. J Clin Oncol. 2012; 30:1796-1804.

8. Liedtke C, Mazouni C, Hess KR, Andre F, Tordai A, Mejia JA, Symmans WF, Gonzalez-Angulo AM, Hennessy B, Green M, Cristofanilli M, Hortobagyi GN and Pusztai L. Response to neoadjuvant therapy and long-term survival in patients with triple-negative breast cancer. J Clin Oncol. 2008; 26:1275-1281.

9. Bardou VJ, Arpino G, Elledge RM, Osborne CK and Clark GM. Progesterone receptor status significantly improves outcome prediction over estrogen receptor status alone for adjuvant endocrine therapy in two large breast cancer databases. J Clin Oncol. 2003; 21:1973-1979.

10. Creighton CJ, Kent Osborne C, van de Vijver MJ, Foekens JA, Klijn JG, Horlings HM, Nuyten D, Wang Y, Zhang Y, Chamness GC, Hilsenbeck SG, Lee AV and Schiff R. Molecular profiles of progesterone receptor loss in human breast tumors. Breast Cancer Res Treat. 2009; 114:287-299.

11. Kuerer HM, Newman LA, Smith TL, Ames FC, Hunt KK, Dhingra K, Theriault RL, Singh G, Binkley SM, Sneige N, Buchholz TA, Ross MI, McNeese MD, Buzdar AU, Hortobagyi GN and Singletary SE. Clinical course of breast cancer patients with complete pathologic primary tumor and axillary lymph node response to doxorubicin-based neoadjuvant chemotherapy. J Clin Oncol. 1999; 17:460469.

12. von Minckwitz G and Martin M. Neoadjuvant treatments for triple-negative breast cancer (TNBC). Ann Oncol. 2012; 23 Suppl 6:vi35-39.

13. Jones RL, Salter J, A'Hern R, Nerurkar A, Parton M, Reis-Filho JS, Smith IE and Dowsett M. The prognostic significance of Ki67 before and after neoadjuvant chemotherapy in breast cancer. Breast Cancer Res Treat. 2009; 116:53-68.

14. Goldstein NS, Decker D, Severson D, Schell S, Vicini F, 
Margolis J and Dekhne NS. Molecular classification system identifies invasive breast carcinoma patients who are most likely and those who are least likely to achieve a complete pathologic response after neoadjuvant chemotherapy. Cancer. 2007; 110:1687-1696.

15. Goldhirsch A, Wood WC, Coates AS, Gelber RD, Thurlimann B, Senn HJ and Panel m. Strategies for subtypes - dealing with the diversity of breast cancer: highlights of the St. Gallen International Expert Consensus on the Primary Therapy of Early Breast Cancer 2011. Ann Oncol. 2011; 22:1736-1747.

16. Issa-Nummer $\mathrm{Y}$, Darb-Esfahani $\mathrm{S}$, Loibl S, Kunz G, Nekljudova V, Schrader I, Sinn BV, Ulmer HU, Kronenwett R, Just M, Kuhn T, Diebold K, Untch M, Holms F, Blohmer JU, Habeck JO, et al. Prospective Validation of Immunological Infiltrate for Prediction of Response to Neoadjuvant Chemotherapy in HER2-Negative Breast Cancer - A Substudy of the Neoadjuvant GeparQuinto Trial. PLoS One. 2013; 8:e79775.

17. Tran B and Bedard PL. Luminal-B breast cancer and novel therapeutic targets. Breast cancer research : BCR. 2011; 13:221.

18. Cornen S, Guille A, Adelaide J, Addou-Klouche L, Finetti P, Saade MR, Manai M, Carbuccia N, Bekhouche I, Letessier A, Raynaud S, Charafe-Jauffret E, Jacquemier J, Spicuglia S, de The H, Viens P, et al. Candidate luminal B breast cancer genes identified by genome, gene expression and DNA methylation profiling. PLoS One. 2014; 9:e81843.

19. Jacquemier J, Charafe-Jauffret E, Monville F, Esterni B, Extra JM, Houvenaeghel G, Xerri L, Bertucci F and Birnbaum D. Association of GATA3, P53, Ki67 status and vascular peritumoral invasion are strongly prognostic in luminal breast cancer. Breast cancer research : BCR. 2009; 11:R23.

20. Feeley LP, Mulligan AM, Pinnaduwage D, Bull SB and Andrulis IL. Distinguishing luminal breast cancer subtypes by Ki67, progesterone receptor or TP53 status provides prognostic information. Modern pathology : an official journal of the United States and Canadian Academy of Pathology, Inc. 2014; 27:554-561.

21. Cancello G, Maisonneuve P, Rotmensz N, Viale G, Mastropasqua MG, Pruneri G, Montagna E, Iorfida M, Mazza M, Balduzzi A, Veronesi P, Luini A, Intra M, Goldhirsch A and Colleoni M. Progesterone receptor loss identifies Luminal B breast cancer subgroups at higher risk of relapse. Ann Oncol. 2013; 24:661-668.

22. Arpino G, Weiss H, Lee AV, Schiff R, De Placido S, Osborne CK and Elledge RM. Estrogen receptor-positive, progesterone receptor-negative breast cancer: association with growth factor receptor expression and tamoxifen resistance. Journal of the National Cancer Institute. 2005; 97:1254-1261.

23. Chen S, Liu Y, Huang L, Chen CM, Wu J and Shao ZM. Lymph node counts and ratio in axillary dissections following neoadjuvant chemotherapy for breast cancer: a better alternative to traditional $\mathrm{pN}$ staging. Ann Surg Oncol. 2014; $21: 42-50$.

24. Eisenhauer EA, Therasse P, Bogaerts J, Schwartz LH, Sargent D, Ford R, Dancey J, Arbuck S, Gwyther S, Mooney M, Rubinstein L, Shankar L, Dodd L, Kaplan R, Lacombe D and Verweij J. New response evaluation criteria in solid tumours: revised RECIST guideline (version 1.1). Eur J Cancer. 2009; 45:228-247.

25. Ogston KN, Miller ID, Payne S, Hutcheon AW, Sarkar TK, Smith I, Schofield A and Heys SD. A new histological grading system to assess response of breast cancers to primary chemotherapy: prognostic significance and survival. Breast. 2003; 12:320-327. 